\title{
Development of an Infusion Based on Romaine Lettuce and Banana for Treating Insomnia
}

\author{
Daniel Revelo-Cáceres ${ }^{1 *}$, María Jesús Oliveras-López ${ }^{2}$, Marina Villalón-Mir ${ }^{1}$, \\ Miguel Navarro-Alarcón ${ }^{1}$, Herminia López-García de la Serrana ${ }^{1}$ \\ ${ }^{1}$ Facultad de Farmacia, Universidad de Granada, Granada, España \\ ${ }^{2}$ Departamento de Biología Molecular e Ingeniería Bioquímica, Universidad Pablo de Olavide, Sevilla, España \\ Email: *danielrevelo1993@hotmail.com
}

How to cite this paper: Revelo-Cáceres, D., Oliveras-López, M.J., Villalón-Mir, M., Navarro-Alarcón, M. and de la Serrana, H.L.-G. (2020) Development of an Infusion Based on Romaine Lettuce and Banana for Treating Insomnia. Agricultural Sciences, 11, 529-539.

https://doi.org/10.4236/as.2020.116033

Received: April 10, 2020

Accepted: May 30, 2020

Published: June 2, 2020

Copyright $\odot 2020$ by author(s) and Scientific Research Publishing Inc. This work is licensed under the Creative Commons Attribution International License (CC BY 4.0).

http://creativecommons.org/licenses/by/4.0/

\begin{abstract}
An infusion was developed based on green romaine lettuce leaves and banana. Further, its sensorial acceptability was evaluated as a possible alternative treatment for insomnia. The romaine lettuces and bananas used were washed, chopped, dehydrated and ground. Optimal lettuce dehydration time was determined through charting the weight lost and examining the curve. This was found after 180 minutes at $60^{\circ} \mathrm{C}$. Two formulas were developed, the first with $1.1 \mathrm{~g}$ of dehydrated romaine lettuce and $0.2 \mathrm{~g}$ of dehydrated banana, and the second with $1.1 \mathrm{~g}$ of dehydrated romaine lettuce and $0.4 \mathrm{~g}$ of dehydrated banana. Sensory analysis was conducted using sorting tests for taste, aroma, paired preferences and intention to purchase. Tasters preferred the formula with a lower quantity of dehydrated banana. $85 \%$ of tasters indicated that they would buy this infusion due to its potential functional properties for overcoming insomnia.
\end{abstract}

\section{Keywords}

Infusion, Romaine Lettuce, Banana, Insomnia, Sensory Analysis

\section{Introduction}

Insomnia is a disorder that is related with low quality sleep patterns. It is very well known given that is affects almost $30 \%$ of the global population [1]. It can easily present in certain circumstances such as when high levels of stress are present, in pregnant women, or in individuals who have recently suffered losses in their family. Likewise, greater trends towards insomnia are seen to exist amongst females and individuals aged older than 65 years [2]. Suffering from this disorder can alter, to a large extent, the daily lives of affected individuals. 
The most commonly seen effects are a decrease in productivity at work, tendencies to have various "siestas" (naps) during the day, diminished academic performance and behavioural problems [3].

It can be seen that research into and treatment of insomnia has gathered pace in recent years and become of great importance. This is due to the fact that insomnia is closely related with cardiovascular diseases [4], allergic rhinitis [5], type 2 diabetes [6] and thyroid disorders [7].

The most heard of utilised medications in the treatment of insomnia are benzodiazepines, anti-depressants and anticonvulsants drugs. Nevertheless, the use of these medications can cause secondary effects such as drowsiness, headaches and a metallic taste in the mouth. In some cases, use may also lead to dependence and a rebound effect of insomnia once use is discontinued [8] [9]. This has led to a surge in various non-pharmacological mechanisms such as sleep hygiene, relaxation therapy, sleep restriction therapy and consumption of foods that help bring about sleep, amongst others. These mechanisms are based on improving the duration and quality of sleep without causing secondary effects, thus providing an advantage over pharmacological mechanisms [3].

A strong interest into the consumption of fruits and medicinal plants which induce sleep has emerged, with consumption of these foodstuffs in their natural or processed forms (extracts, infusions, pills) currently being a common topic. The most known are camomile, valerian, passionflower, kiwi and banana. Despite this, certain vegetables exist which were formerly known for their sedative effects and capacity to induce sleep. An example of this is lettuce; this would be capable of stimulating sleep due to the content of the lactucin component which it possesses in its interior [10].

Lactucin is a sesquiterpene lactone which is present in lettuce and which confers a bitter taste to this vegetable [11]. Lactucin content is not constant in all varieties of lettuce; nonetheless, it has positive health effects [12]. Nevertheless, it has been seen that green leaf romaine lettuce is the species with the highest lactucin content [13]. The mechanism of action of lactucin with regards to sleep disorders acts through the modification of $\gamma$-aminobutyric acid (GABA) levels and those of its receptors [14]. GABA is a neurotransmitter which is responsible for the inhibition-excitation balance of the human brain [15]. Medications that are used to treat insomnia such as benzodiazepines, increase the flow of GABA, enabling people to fall to sleep [16]. In various studies of individuals with depression [17], bipolar disorders [18] and insomnia [19], significantly lower levels of GABA have been observed in comparison to healthy controls.

Rats who were treated with both leaf extract and seeds from green leaf romaine lettuce demonstrated a reduction in sleep latency and an increase in sleep duration, these both being desirable factors in individuals who suffer from insomnia [13]. Albino rats who were treated with pentobarbital and lettuce extract produced similar results, with latency decreasing and sleep increasing to a considerable extent. For this reason, the researchers concluded that lettuce extract 
strengthens the effect of pentobarbital in individuals with sleep disorders [20]. In the same way, use of romaine lettuce combined with Virginia skullcap (Scutellaria lateriflora) managed to increase sleep duration in rats by $50.9 \%$ in comparison to rats treated only with pentobarbital [19]. Pregnant women aged between 20 and 45 years old who suffered insomnia, were administered capsules containing $1000 \mathrm{mg}$ of lettuce seeds. At the end of the research, it was determined that sleep quality had increased. This is of great importance due to the fact that medication use for insomnia can lead to premature births. This supports use of lettuce as a natural remedy to treat this disorder [21]. Likewise, individuals with insomnia who were administered pills containing $1000 \mathrm{mg}$ of lettuce seed oil, achieved high percentage increases in sleep duration [22]. The objective of the present research was to develop an infusion based on green leaf romaine lettuce and banana, evaluating its sensory acceptability as a possible alternative treatment for insomnia.

\section{Materials and Methods}

\subsection{Vegetable Material}

The lettuces and bananas used in the present experiment were acquired from a supermarket in the city of Granada and later transported to laboratories belonging to the Innofood company. Green leaf romaine lettuce was used. The lettuces were received pre-washed, inside plastic packaging and ready for consumption.

The green leaf romaine lettuces were removed from their packaging, and classified according to size, and appearance or absence of defects. After washing the lettuces, the leaves were separated from the lettuce bud and dried at room temperature.

Further, the Cavendish variety of banana was used in its optimal state of maturation. In the same way, the bananas were classified according to size, and the appearance of absence of defects.

\subsection{Dehydration of Lettuces and Bananas}

Washed and dried leaves from the romaine lettuce were chopped and placed on dehydration trays made by the rational brand. A dehydration process was used that occurs in warm air at a temperature of $60^{\circ} \mathrm{C}$, with this temperature being selected in accordance with results obtained in previously conducted studies [13]. During this treatment, the romaine lettuces were weighed every 20 minutes until a constant weight was reached.

In dehydrating the banana, the fruit was peeled and washed, cut into slices and later submerged in a $0.2 \%$ citric acid solution for 30 minutes. The dehydration method used utilised warm air, applying a temperature of $50^{\circ} \mathrm{C}$ over 6 hours [23]. The parameters used in this dehydration procedure were based on results previously obtained in prior analysis [23]. 


\subsection{Infusion Formulation}

Dehydrated romaine lettuce leaves were ground in a Bosch ErgoMixx grinder. The same procedure was carried out with the pieces of dehydrated banana.

Two formulations were used, in both cases the quantity of dehydrated romaine lettuce was $1.1 \mathrm{~g}$, following successful results obtained by various research studies with this quantity [13] [21]. In the case of banana, quantities were selected with the aim of obtaining a pleasant taste and aroma for the consumer.

\subsection{Sensory Analysis}

Sensory analysis was conducted through two types of test; sorting and paired preference. In order to carry out these tests, 60 individuals were required who were given two glasses (formulation 1 and 2) containing $15 \mathrm{ml}$ of the lettuce and banana infusion. Samples were coded with a four-digit code.

In the sorting test, tasters were requested to place the tasted samples in descending order with regards to their attributes of taste and aroma. A similar method was followed for the paired preference test, however, in this analysis tasters selected the sample for which they had the greatest preference and communicated whether they would be willing to buy it.

The results obtained were analysed using tables presenting the minimum number of correct responses in order to establish significance at various levels of probability for preference tests following a binomial distribution. Significance level was set at $\mathrm{p}=0.05$.

\subsection{Packaging}

The sample showing the highest level of acceptance following performance of the sensory analysis was placed in food grade filter paper bags. Following this, packets of 20 bags containing the infusion were formed within cardboard boxes, with the bags being placed in rows.

Finally, a logotype was designed and a name conceived for the romaine lettuce and banana infusion. Throughout this process the best ideas were analysed, which enabled appropriate discovery of the product type and the benefits offered by it. This process was carried out with the objective of achieving potential introduction of the product into the relevant market.

\section{Results}

\subsection{Dehydration of Romaine Lettuce and Bananas}

Romaine lettuce leaves demonstrated a notable reduction in weight during dehydration treatment. At the beginning of the trial the vegetable material had a weight of $500 \mathrm{~g}$, following 180 minutes the lettuce leaves showed a weight of 16.09 g. From this moment onwards, constant weight loss was evidenced which could be seen through the fact that continuous weight measurements were taken over a period of 60 minutes, during which time this parameter did not demon- 
strate any variation.

Weight loss in lettuce leaves is related with the decrease in water content, a behaviour that is characteristic of the vegetable during dehydration treatment. The weight loss seen in romaine lettuce leaves during the dehydration process can be observed in Figure 1.

Romaine lettuce leaves presented a water content of $95.77 \%$ prior to being subjected to the dehydration process, with this measurement being carried out with the help of a Sartorius MA35 thermobalance. Water content of the leaves decreased significantly over time under dehydration conditions, obtaining a mean of $9.43 \%$.

Dehydrating the banana showed that method used did not change bananas' sensory properties. Bananas presented a water content of $81 \%$ prior to being subjected to the dehydration process, water content of the bananas decreased over time under dehydration conditions, obtaining a mean of $13 \%$.

\subsection{Formulation of the Infusion}

The two formulations proposed at the beginning of this research paper, those being elaborated using $1.1 \mathrm{~g}$ of dehydrated romaine lettuce with $0.2 \mathrm{~g}$ and $0.4 \mathrm{~g}$ of dehydrated banana, respectively, had to be modified after conducting an initial sensory test in the Innofood laboratories. This modification was made because it was uncovered that the flavour and aroma of banana in the two formulations could not be easily appreciated. For this reason, it was decided to proceed using scented banana powder ZI-895-665-9, which is property of the Givaudan company.

Table 1 shows the percentages of scented banana powder used in each of the formulations. 7 tests were carried out on both formulation 1 and formulation 2, varying the aroma percentage from $0 \%$ (control) to $3 \%$ (final dosage).

Use of scented banana powder is permitted for both of the formulations as Regulation (CE) $\mathrm{N}^{\circ} 1334 / 2008$ of the European Council and Parliament permits the use of aromas in foodstuffs, in cases that these aromas do not present any

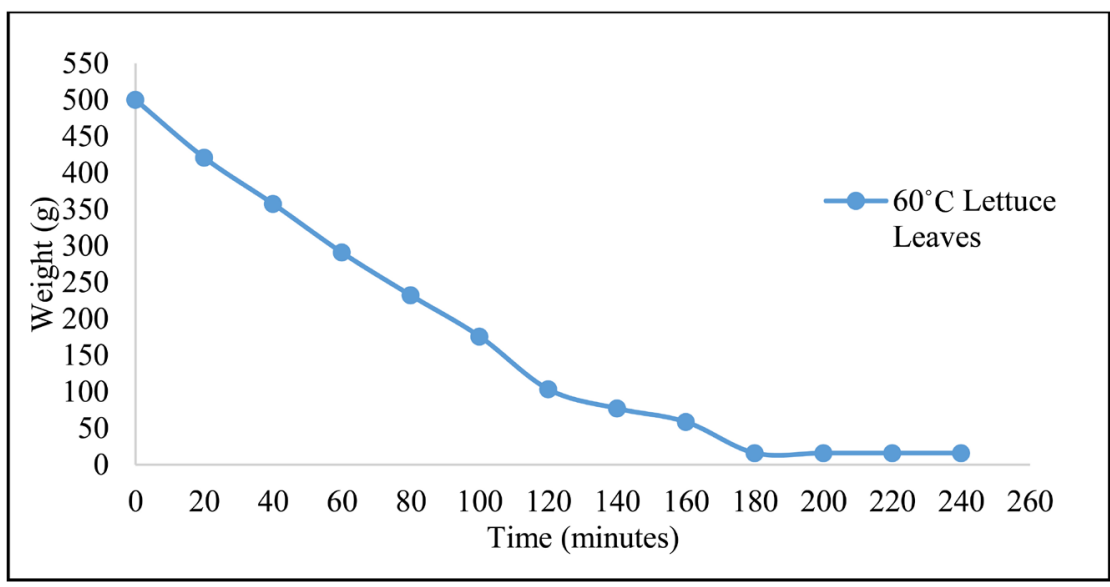

Figure 1. Weight loss curve for romaine lettuce. 
risk to the health of consumers. It is recommended to follow manufacturer instructions with regards to the percentage used [24].

\subsection{Taste Sorting Test}

Figure 2 presents the results of the taste sorting test. These verify that the tasters were able to differentiate the different flavour intensities of the two formulations. This can be seen in the way that $57 \%$ of tasters considered that formulation 2 had a stronger taste relative to formulation 1 . This appreciation is related with the quantity of banana used, given that formulation 2 contained twice as many grams of banana as formulation 1 .

\subsection{Aroma Sorting Test}

Through the aroma sorting test similar behaviours were evidenced to those presented in the taste test. Figure 3 shows that 55\% of tasters identified a greater

Table 1. Use of scented banana powder.

\begin{tabular}{cccccccc}
\hline & \multicolumn{2}{c}{ Formulation 1 } & \multicolumn{5}{c}{ Formulation 2 } \\
\hline Sample & Lettuce (g) & Banana (g) & Aroma (\%) & Sample & Lettuce (g) & Banana (g) Aroma (\%) \\
\hline Control & 1.1 & 0.2 & 0 & Control & 1.1 & 0.4 & 0 \\
1 & 1.1 & 0.2 & 0.1 & 1 & 1.1 & 0.4 & 0.1 \\
2 & 1.1 & 0.2 & 0.2 & 2 & 1.1 & 0.4 & 0.2 \\
3 & 1.1 & 0.2 & 0.3 & 3 & 1.1 & 0.4 & 0.3 \\
4 & 1.1 & 0.2 & 0.5 & 4 & 1.1 & 0.4 & 0.5 \\
5 & 1.1 & 0.2 & 1 & 5 & 1.1 & 0.4 & 1 \\
6 & 1.1 & 0.2 & 2 & 6 & 1.1 & 0.4 & 2 \\
7 & 1.1 & 0.2 & 3 & 7 & 1.1 & 0.4 & 3
\end{tabular}

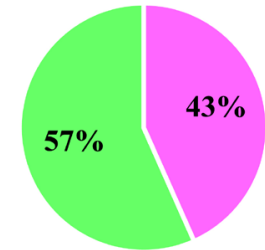

- Formulation $1 \quad$ Formulation 2

Figure 2. Taste sorting test.

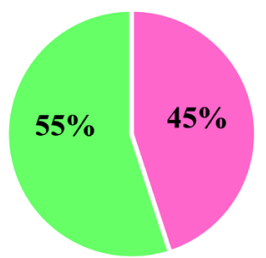

- Formulation 1 Formulation 2

Figure 3. Aroma sorting test. 
aroma intensity in formulation 2 . Nevertheless, this rating is not likely to be explained solely by the quantity of banana used but also by the use of scented banana powder.

\subsection{Paired Preference Test}

Figure 4 shows the results of the paired preference test. From the data obtained it can be identified that 43 out of the 60 tasters chose formulation 1, or in other words, tasters preferred the sample with a lower intensity of banana flavour and aroma. The 43 positive responses given towards formulation 1 exceeded the minimum number of correct responses required to establish significance (39 affirmative responses). From this, it could be determined that this formulation was ideal for the elaboration of a romaine lettuce and banana infusion.

In the case of the survey of intentions to purchase the product, tasters who would be willing to purchase formulation 1 were found to be in the same group as tasters who were willing to purchase formulation 2. A similar treatment was applied to those who would not be willing to purchase this infusion. As can be observed in Figure 5, 85\% of tasters showed a strong interest in acquiring this infusion due to its potential properties for tackling insomnia.

The $15 \%$ of individuals who stated that they would not be willing to buy this product remarked that the decision was based on their low consumption of products available from the infusion market as opposed to any sensory response. Indeed, they considered that the product presented excellent organoleptic properties.

\subsection{Label Design}

The colours black and green were used in the label design due to the fact that these colours give the impression of tranquillity and relaxation. LactuSleep was

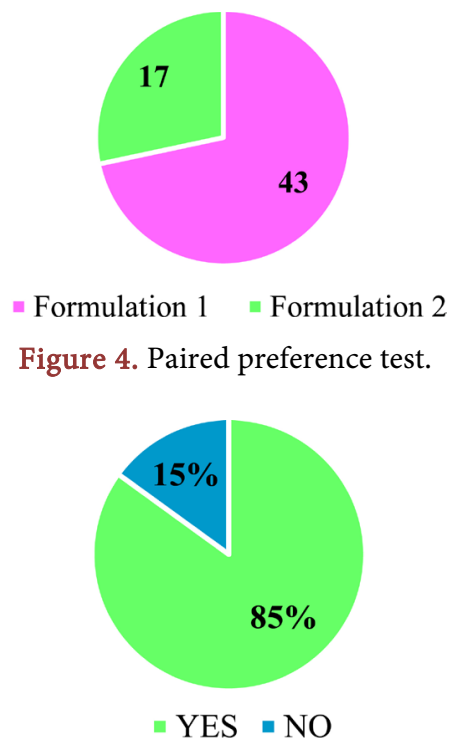

Figure 5. Results from the intention to purchase test. 
the name given to the infusion. The word Lactu alludes to the scientific name Lactuca and Sleep which refers to the power of the infusion for inducing sleep. Figure 6 presents the label developed for this product.

\subsection{Final Product}

The sensory analysis conducted enabled it to be determined that formulation 1 was more acceptable to tasters and for this reason it was selected for elaboration of the final product. From this, we proceeded to fill food grade filter paper bags with $1.1 \mathrm{~g}$ of dehydrated romaine lettuce, $0.2 \mathrm{~g}$ dehydrated banana and $3 \%$ of scented banana powder.

Packs of 20 units of these small bags were then placed inside cardboard boxes, with a label with the name "LactuSleep" being located on the extreme corner of each bag. Likewise, nutritional information for the infusion was developed, as can be seen in Table 2 .

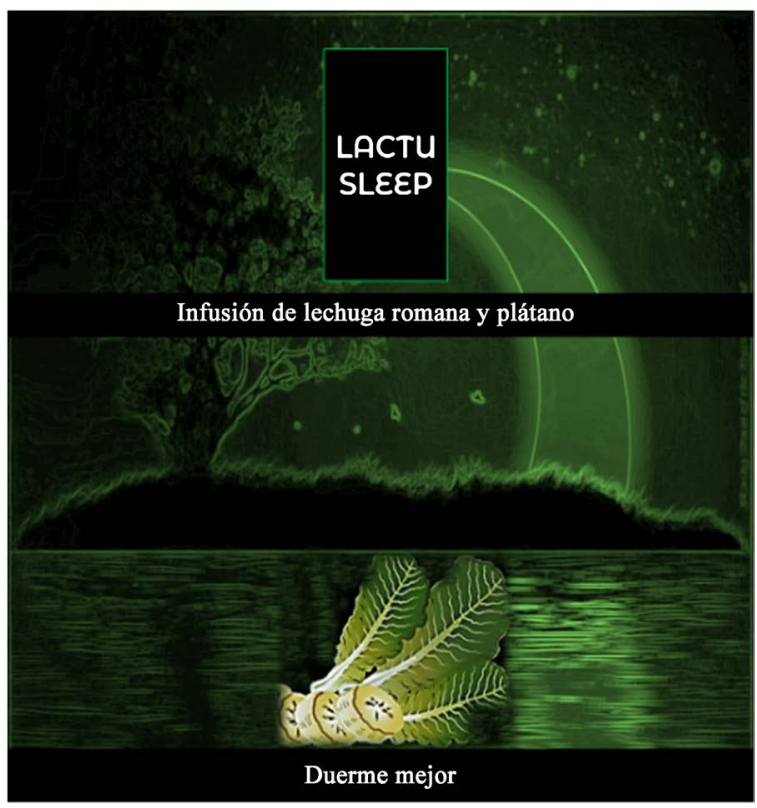

Figure 6. Label for the LactuSleep infusion.

Table 2. Nutritional information for the infusion.

\begin{tabular}{cc} 
Nutritional Information \\
Mean values per 100 ml of infusion \\
\hline Energy value (calories) \\
Fats: & $4 \mathrm{KJ} / 1 \mathrm{Kcal}$ \\
Saturated & $0 \mathrm{~g}$ \\
Carbohydrates: & $0 \mathrm{~g}$ \\
Sugars & $0.1 \mathrm{~g}$ \\
Dietary fibre & $0 \mathrm{~g}$ \\
Protein & $0 \mathrm{~g}$ \\
Salt & $0 \mathrm{~g}$ \\
\hline
\end{tabular}




\section{Conclusion}

The measurement of weight loss in romaine lettuce leaves enabled determination of the suitable conditions for dehydration treatment, these being $60^{\circ} \mathrm{C}$ over a period of 180 minutes. These conditions do not cause any damage to the vegetable material. Banana use as an agent for improving the sensory characteristics of the romaine lettuce infusion was found to be successful. This was due to the fact that outcomes of sorting tests for both flavour and aroma showed that this fruit was easily identified, whilst also receiving good acceptability scores on behalf of tasters. The paired preference test showed that 43 of the 60 tasters preferred the formulation that contained a lower quantity of dehydrated banana and for this reason it was used to elaborate the final product. The survey of intentions to purchase revealed that $85 \%$ of tasters were willing to purchase this infusion due to its functional properties in relation to insomnia. This percentage enables prediction of the potential success of this product in the infusion market.

\section{Acknowledgements}

We express our gratitude to the InnoFood company located in la Zubia, Granada, Spain, for their large contribution to elaboration of this infusion.

\section{Conflicts of Interest}

The authors declare no conflicts of interest regarding the publication of this paper.

\section{References}

[1] Stavropoulos, K., Imprialos, K., Doumas, M., Karagiannis, A. and Papademetriou, V. (2019) Insomnia and Hypertension: A Misty Landscape. The Journal of Clinical Hypertension, 21, 835-837. https://doi.org/10.1111/jch.13519

[2] Dollander, M. (2002) Etiology of Adult Insomnia. Literature Review in L'Encéphale, 28, 493-502.

[3] Pradeep, C. and Harleen, M. (2019) Sleep Medicine: Insomnia and Sleep. Missouri Medicine, 116, 68-75.

[4] Javaheri, S. and Redline, S. (2017) Insomnia and Risk of Cardiovascular Disease. Chest, 152, 435-444. https://doi.org/10.1016/j.chest.2017.01.026

[5] Lin, Y., Lai, C. and Chien, C. (2017) Is Insomnia a Risk Factor for New Onset Asthma? A Population-Based Study in Taiwan. BMJ Open, 7, e018714.

https://doi.org/10.1136/bmjopen-2017-018714

[6] Kawakami, N., Takatsuka, N. and Shimizu, H. (2004) Sleep Disturbance and Onset of Type 2 Diabetes. Diabetes Care, 27, 282-283. https://doi.org/10.2337/diacare.27.1.282

[7] Xia, L., Chen, G., Li, Z., Jiang, S. and Shen, J. (2013) Alterations in Hypothalamus-Pituitary-Adrenal/Thyroid Axes and Gonadotropin-Releasing Hormone in the Patients with Primary Insomnia: A Clinical Research. PLoS ONE, 8, e71065. https://doi.org/10.1371/journal.pone.0071065

[8] Nutt, D. and Stahl, S.M. (2010) Searching for Perfect Sleep: The Continuing Evolution 
of GABAA Receptor Modulators as Hypnotics. Journal of Psychopharmacology, 24, 1601-1612. https://doi.org/10.1177/0269881109106927

[9] Becker, P. and Somiah, M. (2015) Non-Benzodiazepine Receptor Agonists for Insomnia. Sleep Medicine Clinics, 10, 57-76.

https://doi.org/10.1016/j.jsmc.2014.11.002

[10] Kim, M., Moon, Y., Tou, J., Mou, B. and Waterland, N. (2016) Nutritional Value, Bioactive Compounds and Health Benefits of Lettuce (Lactuca sativa L.). Journal of Food Composition and Analysis, 49, 19-34. https://doi.org/10.1016/j.jfca.2016.03.004

[11] Wesolowska, A., Nikiforuk, A., Michalska, K., Kisiel, W. and Chojnacka-Wójcik, E. (2006) Analgesic and Sedative Activities of Lactucin and Some Lactucin-Like Guaianolides in Mice. Journal of Ethnopharmacology, 107, 254-258.

https://doi.org/10.1016/j.jep.2006.03.003

[12] Giambanelli, E., D’Antuono, F., Ferioli, F., Garrido, A. and Romero-González, R. (2018) Sesquiterpene Lactones and Inositol 4-Hydroxyphenylacetic Acid Derivates in Wild Edible Leafy Vegetables from Central Italy. Journal of Food Composition and Analysis, 72, 1-6. https://doi.org/10.1016/j.jfca.2018.06.003

[13] Dum Kim, H., Hong, K.-B., Noh, O.N. and Joo Suh, H. (2017) Sleep-Inducing Effect of Lettuce (Lactuca sativa) Varieties on Pentobarbital-Induced Sleep. Food Science and Biotechnology, 26, 807-814. https://doi.org/10.1007/s10068-017-0107-1

[14] Chen, L., Chen, I., Wang, B. and Shao, C. (2009) Drug-Use Pattern of Chinese Herbal Medicines in Insomnia: A 4-Year Survey in Taiwan. Journal of Clinical Pharmacy and Therapeutics, 34, 555-560. https://doi.org/10.1111/j.1365-2710.2009.01038.x

[15] Xu, G., Broadbelt, K. and Haynes, R. (2011) Late Development of the GABAergic System in the Human Cerebral Cortex and White Matter. Journal of Neuropathology \& Experimental Neurology, 70, 841-858. https://doi.org/10.1097/NEN.0b013e31822f471c

[16] Ramakrishnan, K. and Scheid, D. (2007) Treatment Options for Insomnia. American Family Physician, 76, 517-526.

[17] Gold, B., Bowers, M., Roth, R. and Sweeney, P. (1980) GABA Levels in CSF of Patients with Psychiatric Disorders. American Journal of Psychiatry, 137, 362-364. https://doi.org/10.1176/ajp.137.3.362

[18] Mann, J., Oquendo, M. and Watson, K. (2014) Anxiety in Major Depression and Cerebrospinal Fluid Free Gamma-Aminobutyric Acid. Depression and Anxiety, 31, 814-821. https://doi.org/10.1002/da.22278

[19] Hong, K., Han, S., Park, Y., Suh, H. and Choi, H. (2018) Romaine Lettuce/Skullcap Mixture Improves Sleep Behavior in Vertebrate Models. Biological and Pharmaceutical Bulletin, 41, 1269-1276. https://doi.org/10.1248/bpb.b18-00267

[20] Ghorbani, A., Rakhshandeh, H. and Reza-Sadeghnia, H. (2013) Potentiating Effects of Lactuca sativa on Pentobarbital-Induced Sleep. Iranian Journal of Pharmaceutical Research, 12, 401-406.

[21] Pour, Z., Hosseinkhani, A., Asadi, N., Shahraki, H., Vafaei, H., Kasraeian, M. and Faraji, A. (2018) Double-Blind Randomized Placebo-Controlled Trial on Efficacy and Safety of Lactuca sativa L. Seeds on Pregnancy-Related Insomnia. Journal of Ethnopharmacology, 227, 176-180. https://doi.org/10.1016/j.jep.2018.08.001

[22] Yakoot, M., Helmy, S. and Fawal, K. (2011) Pilot Study of the Efficacy and Safety of Lettuce Seed Oil in Patients with Sleep Disorders. International Journal of General Medicine, 4, 451-456. https://doi.org/10.2147/IJGM.S21529 
[23] Kanchana, S. and Wickramarachchi, L. (2005) Preservation of Fiber-Rich Banana Blossom as a Dehydrated Vegetable. Science Asia, 31, 265-271. https://doi.org/10.2306/scienceasia1513-1874.2005.31.265

[24] EUR-Lex (2008) Reglamento (CE) N 1334/2008 del Parlamento Europeo y del Consejo sobre los aromas y determinados ingredientes alimentarios con propiedades aromatizantes utilizados en los alimentos. 